\title{
Ultra Low Dispersion Spectroscopy with Gaia and Astronomical Slitless Surveys
}

\author{
R. Hudec, L. Hudec, M. Klíma
}

\begin{abstract}
The ultra-low dispersion spectroscopy to be applied in the ESA Gaia space observatory and the ground-based objectiveprism plate surveys represent a similar type of astrophysical data. Although the dispersion in plate surveys is usually larger than in the Gaia blue and red photometers (BP/RP), the spectral resolutions differ by a factor of $2-3$ only, since the resolution in ground-based spectra is seeing-limited. We argue that some of the algorithms developed for digitized objective-prism plates can also be applied for the Gaia spectra. At the same time, the plate results confirm the feasibility of observing strong emission lines with Gaia RP/BP.
\end{abstract}

Keywords: astronomical spectroscopy, low dispersion spectroscopy, slitless spectroscopy, Gaia.

\section{Introduction}

The ESA Gaia is a primary astrometry ESA mission to be launched in 2013. The satellite payload consists of a single integrated instrument, the design of which is characterised by a dual telescope concept with a common structure and a common focal plane ${ }^{1}$. Both telescopes are based on a three-mirror anastigmat design. Beam combination is achieved in image space with a small beam combiner. Silicon-carbide ultra-stable material is used for the mirrors and the telescope structure. There will be a large common focal plane with an array of 106 CCDs. The large focal plane also includes areas dedicated to the spacecraft's metrology and alignment measurements. Three instrument functions/modes are designed: (i) Astrometric mode for accurate measurements, even in densely populated sky regions of up to 3 million stars $/ \operatorname{deg}()$, (ii) Photometric mode based on low-resolution, dispersive spectrophotometry using Blue and Red Photometers (BP and RP) for continuous spectra in the $330-1000 \mathrm{~nm}$ band for astrophysics and chromaticity calibration of the astrometry (Jordi and Carrasco, 2007), and (iii) Spectrometroscopic (RVS) mode for high resolution, with grating, covering a narrow band: $847-874 \mathrm{~nm}$.

The expected limiting magnitude is 20 in photometric mode ${ }^{2}$. In this contribution, we discuss the data expected to be provided by the $\mathrm{BP} / \mathrm{RP}$ photometers, and show that they will represent ultra-low dispersion spectra which can be used in various astrophysical projects. We compare this data with analogous data provided by plate surveys.

\section{Gaia photometers and simulations}

In this paper we focus on the "photometric mode" $\mathrm{RP} / \mathrm{BP}$. Use of the dispersive element (prism) generates ultra-low dispersion spectra. One disperser, called BP for Blue Photometer, operates in the 330-660 nm wavelength range; the other, called RP for Red Photometer, covers the $650-1000 \mathrm{~nm}$ wavelength range. The dispersion is higher at short wavelengths, and ranges from 4 to $32 \mathrm{~nm} /$ pixel for $\mathrm{BP}$ and from 7 to $15 \mathrm{~nm} /$ pixel for $\mathrm{RP}^{3}$. It should be noted however that the photometric CCDs are located at the edge of the focal plane, where the quality of the images is more sensitive to aberrations than astrometric images (Straizys et al., 2010).

The BP and RP spectra will be binned on-chip in the across-scan direction; no along-scan binning is foreseen. RP and $\mathrm{BP}$ will be able to reach object densities on the sky of at least 750000 objects $\mathrm{deg}^{-2}$. The obtained complex images can be simulated by the GIBIS simulator (Figure 1). GIBIS is a pixel-level simulator of the Gaia mission intended to simulate how the Gaia instruments will observe the sky, using realistic simulations of the astronomical sources and of the instrumental properties. It is a branch of the global Gaia Simulator (GaiaSimu) under development within Gaia Coordination Unit 2: Data Simulations.

\footnotetext{
${ }^{1}$ http://sci.esa.int/gaia/, 2011

${ }^{2}$ http://sci.esa.int/gaia/, 2011

${ }^{3}$ http://sci.esa.int/gaia/, 2011
} 

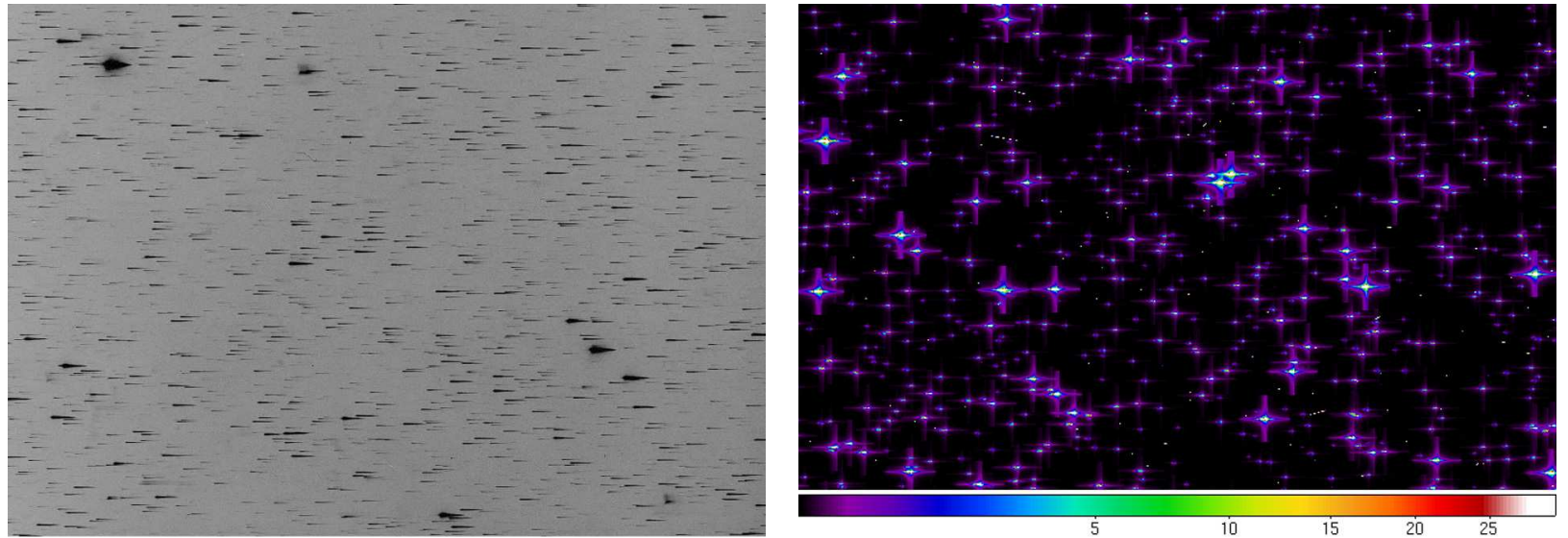

Fig. 1: Left: The prism astronomical survey plate with 1.8 degree prism (Case/PARI), Right: The RP image simulated by the Gaia GIBIS simulator (visualization DS9). This simulated image illustrates the image wings mentioned by Straizys et al., 2006
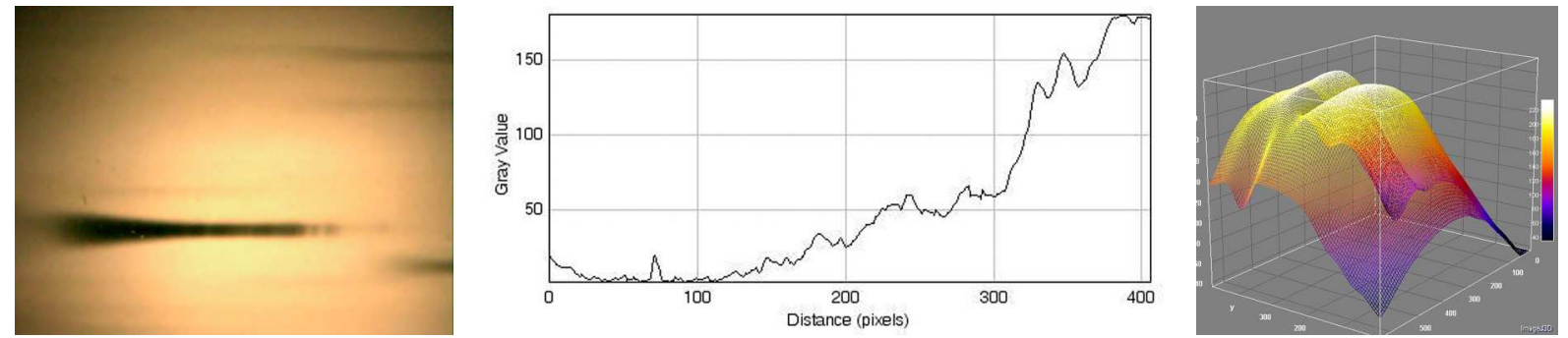

Fig. 2: Image analyses of objects on low dispersion spectroscopic plates of the La Pas German Bolivia southern sky survey. Digitised (using a USB microscope) star image (left), the spectrum (2D) plot (centre), and the 3D plot (right). The $3 \mathrm{D}$ plot allows some details to be studied that are not included in the $2 \mathrm{D}$ plot, such as the image distorsion (image wings) caused by the optics that are used

\section{Ultra low dispersion spectral plate databases}

Low Dispersion Spectroscopy (LDS) astrophysics was evolved and performed at numerous observatories between ca 1910 and 1980. In our project we have analysed the oldest LDS plates at the Carnegie Observatories, in Pasadena, CA, USA. These LDS plates were taken in 1909 with excellent quality, albeit limited FOV. Mostly LDS with Schmidt telescopes (plates with objective prism) were used for various projects e.g. QSO, emission line and Halpha surveys, star classifications, etc.,though some LDS surveys were performed with refractors. This technique was however little used after 1980 . The plate databases were previously mostly evaluated only by manual methods, hence the application of advanced computer methods to this data can yield many new (and probably unexpected) results.

Some of these surveys are listed below (the dispersion data is given in the next section, and we also note that many other similar surveys exist):

(1) Schmidt Sonneberg Camera. Sky survey (selected fields) with a 50/70 cm Schmidt telescope. No online access yet, but the scans can be provided upon request (http://www.stw.tu-ilmenau.de/).

(2) Bolivia Expedition Spectral Plates. These plates offer homogeneous but not full coverage (90 southern Kapteyn's Selected Areas Nos. 116-206 were covered with plates representing $10 \times 10 \mathrm{grad}$ each, hence 9000 square degrees in total) of the southern sky with spectral and direct plates, directed by the Potsdam Observatory. The plates are stored at the Sonneberg Observatory (http://www.stw.tuilmenau.de/) and were taken between 1926-1928, in total about 70000 prism spectra were estimated and published in Potsdam Publications, see Becker (1929) and following papers. See Figure 2 for an example of this type of LDS data.

(3) Hamburg Quasar Survey. A wide-angle objective prism survey searching for quasars with $B<17.5$ in the northern sky. The survey plates were taken with the former Hamburg Schmidt telescope, located at Calar Alto/Spain since 1980. Online access (http://http://www.hs.unihamburg.de/DE/For/Exg/Sur/index.html).

(4) Byurakan Survey. The Digitized First Byurakan Survey (DFBS) is the digitized version of the First Byurakan Survey (FBS). It is the 


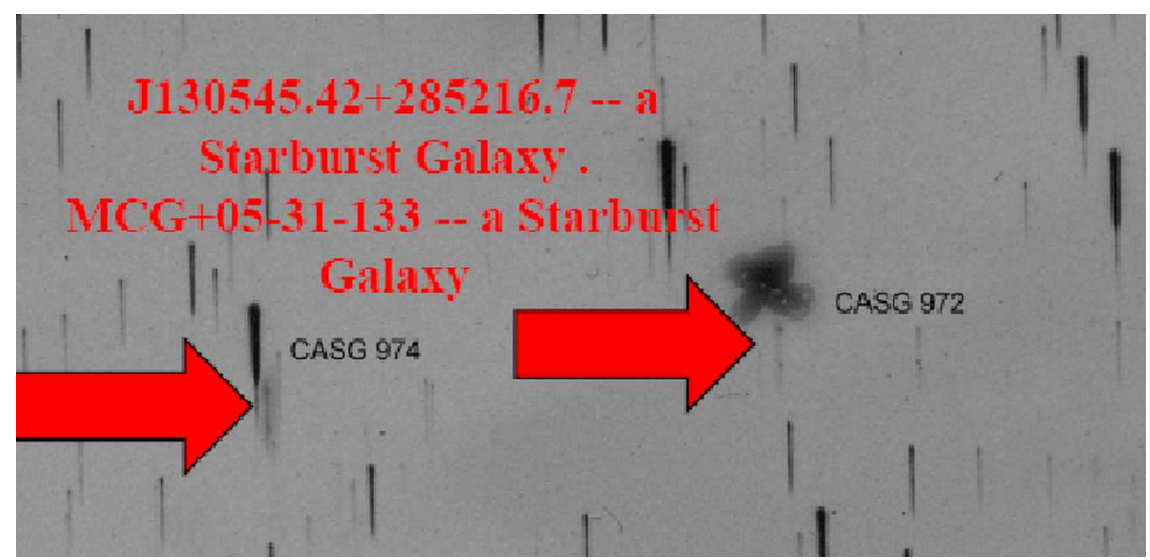

Fig. 3: LDS spectra of two starburst galaxies on PARI Institute plates

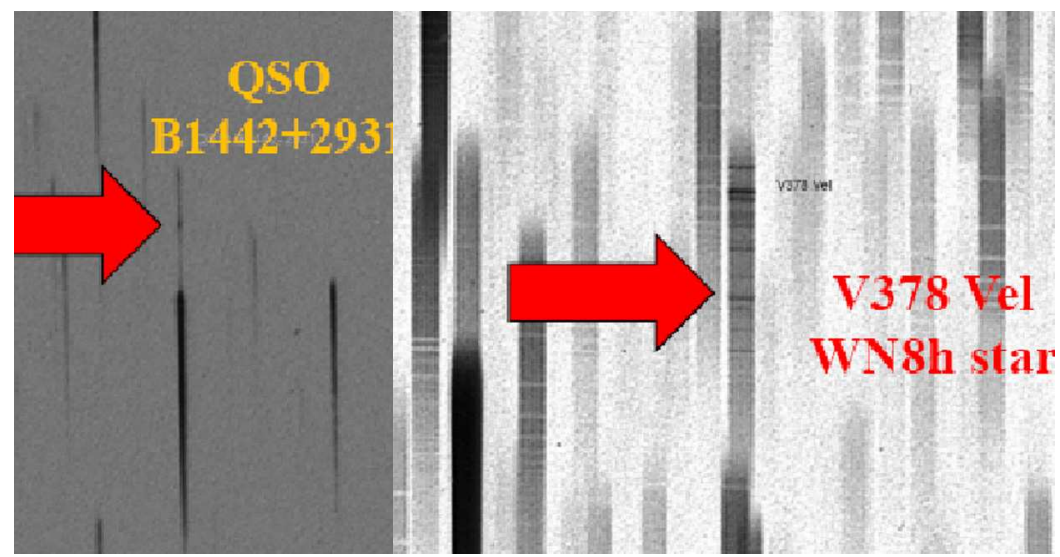

Fig. 4: Examples of objects with prominent spectral features on LDS spectra, PARI Institute plates. Left: QSO, Right: WN8h star V378 Vel. The image on the right is an example of LDS with higher spectral resolution. For these plates numerous emission and absorption lines are visible and various algorithmes are to be developed and applied

largest spectroscopic database in the world, providing low dispersion spectra for 20000000 objects on 1139 FBS fields $=17,056 \mathrm{deg}^{-2}$. Online access (http://byurakan.phys.uniroma1.it/). Sky coverage: $\mathrm{DEC}>-15 \mathrm{deg}$, all RA (except the Milky Way). The prism spectral plates were taken by the $1 \mathrm{~m}$ Schmidt telescope. Limiting magnitude: 17.5 in V. Spectral range: $340-690 \mathrm{~nm}$, spectral resolution $5 \mathrm{~nm}$.

(5) Spectral survey plates in the Astronomical Photographic Data Archive (APDA) located at the Pisgah Astronomical Research Institute (PARI), USA, e.g. Case QSO-Survey (http://www.pari.edu/library). Telescope: $61 / 91 \mathrm{~cm}$ Burrell Schmidt at Kitt Peak, $1.8 \mathrm{deg}$ prism, plate FOV: 5-degree by 5-degree, limiting B magnitude: 18, emulsion: IIIaJ Baked, spectral range: $330 \mathrm{~nm}$ to $530 \mathrm{~nm}$ (Figures 3, 4).

(6) Karl Henize H-alpha plate collection (located since 2010 at PARI) — Michigan-Mount Wilson Southern H-alpha survey (Henize, 1954). A newly (in 2010) re-discovered highly valuable plate collection. 290 high quality plates $15 \times 15$ inches taken in
1950-1952 in South Africa by dedicated telescope by Karl Henize. Telescope aperture D25 cm, dispersion $45 \mathrm{~nm} / \mathrm{mm}$ at Halpha, various filters used (Henize, 1954).

The spectral dispersion of Gaia BP/RP was predetermined with no chance to interfere with the requirements of the scientific community. An important question is whether the dispersion of these devices is sufficient to detect and to study bright spectral features/emission lines. This question was answered e.g. by the extended work of US astrophysicist and NASA astronaut Karl Henize, who spent a large part of his scientific career on low dispersion spectroscopy with an objective prism. We have found the original low dispersion spectral plates that he took about 60 years ago in South Africa and we have analysed them extensively. We found and investigated these plates (probably the complete Henize collection) in the PARI (Pisgah Astronomical Research Institute) Institute, NC, USA. The plates show numerous examples of objects with very prominent and very wide emission lines, which he found in a very extended time-consuming and laborious project. 


\section{Ultra low dispersion images by Gaia RP/BP: algorithms and a comparison with plate surveys}

The algorithms for automated analyses of digitised spectral plates were developed by computer science students (e.g. Hudec, 2007). The main goals are as follows: automated classification of spectral classes, searches for spectral variability (both continuum and lines), searches for objects with specific spectra, correlation of spectral and light changes, searches for transients, and application for Gaia. The archival spectral plates taken with the objective prism offer the possibility to simulate the Gaia low dispersion spectra and related procedures such as searches for spectral variability and variability analyses based on spectro-photometry. We focus on the sets of spectral plates of the same sky region covering long time intervals with good sampling; this enables simulation of the Gaia BP/RP outputs. The main task is automatic classification of stellar objective prism spectra on digitised plates, a simulation and a feasibility study for the low dispersion Gaia spectra. The algorithms that have been developed and tested include the application of novel approaches and techniques with emphasis on neural networks for automated recognition of spectral types of stars, comparing them with atlas spectra. This technique differs from techniques discussed before (e.g. Christlieb et al., 2002, or Hagen et al., 1995). For the future, we plan to continue developing innovative dedicated image processing methods to continue our participation in data extraction and evaluation by providing expertise in the high level image processing with a focus on solving problems of data processing and data extraction emerging from the peculiar way that Gaia is functioning. The expertise available at the Department of Radioelectronics of the CTU Faculty of Electrical Engineering will be further used and developed in this direction.

As illustrated in Figure 1, the Gaia BP/RP and LDS astronomical plates represent similar databases. The motivation for studies comparing these two databases is as follows: (1) A comparison of the simulated Gaia BP/RP images with those obtained from digitized Schmidt spectral plates (both using dispersive elements) for 8 selected test fields, and (2) A feasibility study for application for the algorithms developed for the plates for Gaia. Dispersion is an important parameter, and is discussed later: (1) Gaia BP: 4-32 nm/pixel i.e. $400-3200 \mathrm{~nm} / \mathrm{mm}, 9 \mathrm{~nm} /$ pixel i.e. $\quad 900 \mathrm{~nm} / \mathrm{mm}$ at $\mathrm{H} \gamma, \mathrm{RP}: 7-15 \mathrm{~nm} /$ pixel i.e. $700-1500 \mathrm{~nm} / \mathrm{mm}$. PSF FWHM $\sim 2$ px i.e. spectral resolution is $\sim 18 \mathrm{~nm},(2)$ Schmidt Sonneberg Plates (typical mean value): the dispersion for the
7 deg prism $10 \mathrm{~nm} / \mathrm{mm}$ at $\mathrm{H} \gamma$, and $23 \mathrm{~nm} / \mathrm{mm}$ at $\mathrm{H} \gamma$ for the 3 deg prism. (3) Bolivia Expedition plates: $9 \mathrm{~nm} / \mathrm{mm}$, with calibration spectrum, (4) Hamburg QSO Survey: $1.7 \mathrm{deg}$ prism, $139 \mathrm{~nm} / \mathrm{mm}$ at $\mathrm{H} \gamma$, spectral resolution of $4.5 \mathrm{~nm}$ at $\mathrm{H} \gamma$, (5) Byurakan Survey: $1.5 \mathrm{deg}$ prism, $180 \mathrm{~nm} / \mathrm{mm}$ at $\mathrm{H} \gamma$, resolution $5 \mathrm{~nm}$ at $\mathrm{H} \gamma$ and (6) PARI prism dispersion: $150-340 \mathrm{~nm}$ at $450 \mathrm{~nm}$. We see that the Gaia $\mathrm{BP} / \mathrm{RP}$ dispersion is $\sim 5$ to 10 times less than the dispersion of a typical digitised spectral prism plate, and the spectral resolution of Gaia is $\sim 3$ to 4 times less than for the plates. Note that for plates the spectral resolution is seeing-limited, hence the values represent the best values, and on the plates affected by not superior seeing the spectral resolution is only $\sim 2$ times better when compared to Gaia BP/RP.

\section{Astrophysics with Gaia RP/BP spectro-photometry and LDS}

In our oppinion, the major strength of Gaia for many scientific fields will be in spectro-photometry, as the low dispersion spectra may be transferred to numerous well-defined color filters. As an example, the Optical Afterglows (OAs) of Gamma-Ray Bursts (GRBs) are known to exhibit quite specific color indices, distiguishing them from other types of astrophysical objects (Simon et al., 2001 and 2004a, 2004b), hence a reliable classification of OAs of GRBs will be possible, in principle, using this method. The colors of microquasars may serve as another example: they display blue colors, with a trend of a diagonal formed by the individual objects. This method can be used even for optically faint, and hence distant objects.

The Gaia BP/RP LDS will also provide direct valuable inputs for various fields of recent astrophysics.

Figure 5 illustrates one of the examples, namely the value of LDS for analyses of OAs of GRBs. The emphasis is not only of the LDS spectral continuum profile (reflecting the synchrotron radiation) but also on a study of wide redshiftet Lyman alpha breaks.

The Gaia data will be supported by ground-based optical data with emphasis on robotic telescopes. This is part of the sub-workpackage supplementary optical observation in workpackage Specific Object Studies in the framework of CU7 Unit of Gaia. While this support will focus on supplementay photometry, we have also developed and tested methods involving the fast response LDS (Figure 6). This has scientific justification, as recently the LDS of OAs of GRBs are mostly delayed by 1-10 hours (Fynbo et al., 2009), hence they represent the afterglow optical emission, not the prompt optical emission. 


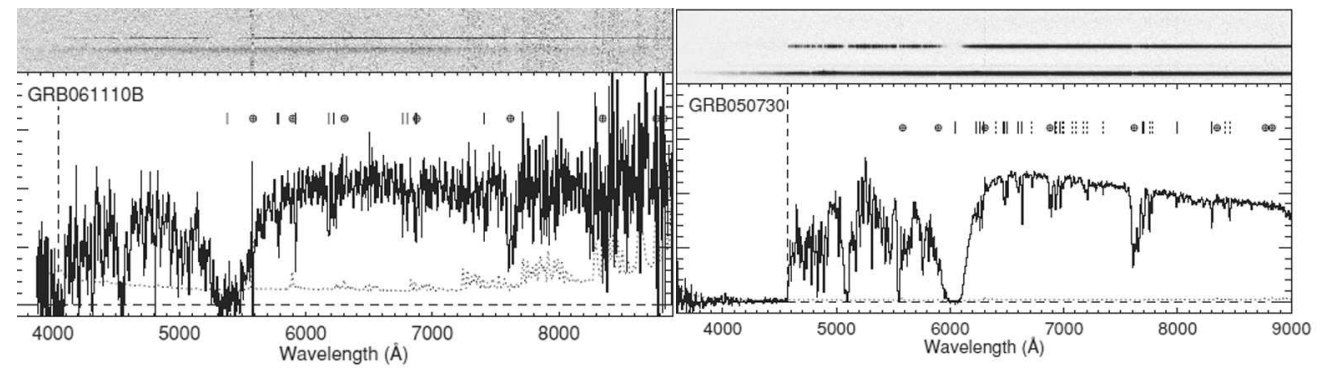

Fig. 5: Examples of LDS of OAs of GRBs with Strong Intervening Absorbers (Fynbo et al., 2009). Evidently the wide redshifted Lyman alpha break will be observable by Gaia RP, and as a consequence there will be a possibility to study highly redshifted objects with Gaia RP up to $z \sim 7$

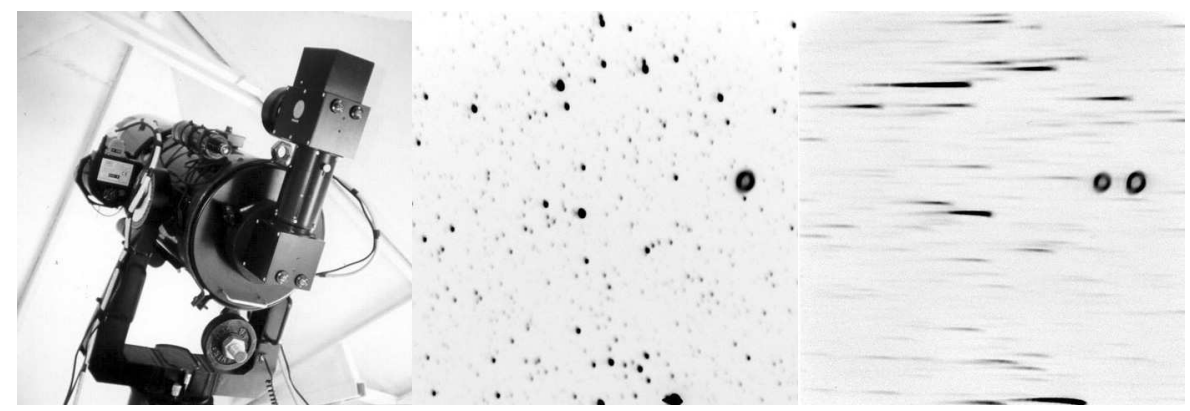

Fig. 6: BOOTES WFS (with prism) constructed by the mechanical shop, Ondrejov Astronomical Institute (left) and the direct image (centre) and prism image (right). Direct Vision Prism is mounted on a $0.3 \mathrm{~m} \mathrm{f} / 10$ telescope, FOV $43^{\prime} \times 28^{\prime}$, dispersion $\sim 4 \AA /$ pixel at $4000 \AA, \sim 30 \AA /$ pixel at $5500 \AA$, and $\sim 100 \AA /$ pixel at $8000 \AA$, limiting magnitude 13.5 in $30 \mathrm{~s}$ (Spectrograph Mode)

The correct color indices however cannot be calculated without careful decontamination of the $\mathrm{BP} / \mathrm{RP}$ spectra (Straizys et al., 2006, Straizys et al., 2010). The energy redistribution effect in the Gaia BP and RP spectra arising from contamination by wings of the image profiles was mentioned and investigated by Straizys et al. (2006), Montegriffo et al. (2007), and Montegriffo (2009). According to these researchers, the Gaia spectra may be used for classifying stars either after applying contamination corrections or by using standard calibration stars with known physical parameters and observed with the Gaia spectrophotometers. In the latter case, there is no way to calculate the real spectral energy distributions, magnitudes, color indices, color excesses or other photometric quantities. The classification has to be made by matching the observed pseudo-energy distributions of the target and the standard stars, or by using pattern recognition algorithms (template matching) over the whole spectrum to estimate the astrophysical parameters of stars.

In addition, Gaia may be useful in the study of strong spectral time variations. It is known that certain types of variable stars (VS) such as Miras, Cepheids, and a few cases of other stars, mostly peculiar variables, exhibit large variations in their spectral types. This field is, however, little exploited, as these studies used to be very laborious (plates were mostly visually inspected) and limited, and hence no review on the spectral variability among VS exists. The ESA Gaia is expected to deliver data to fill this gap.

\section{Recent Results}

Recently, we have digitised the full collection of Henize plates (southern MtWilson-Michigan H-alpha sky survey) and have found and analysed the Northern Mt Wilson-Michigan H-alpha sky survey plates deposited at Carnegie Observatories in Pasadena, CA, USA. Selected plates from this collection have been digitized. The southern sky La Paz Bolivia Expedition survey was recently fully scanned and deposited at Sonneberg Observatory, Germany. In addition, LDS plates located at various observatories (e.g. Sonneberg Schmidt, KPNO, Lick, Mt Hamilton, etc.) were investigated and some of them have been digitized. A technique for on site plate scanning (using a transportable digitization device) was developed and tested. This proved to be essential, as many of the large plate collections do not have any suitable plate scanner.

For the LDS plates deposited at PARI, an extended literature and in situ plate search was carried 
out in order to correlate the literature records and the plates, and also to re-discover and re-investigate various objects with prominent spectral lines using modern methods, with emphasis on objects described many years ago in the literature. Some examples are illustrated in Figures 3 and 4. Advanced investigation and visualization methods were also exploited, with examples shown in Figure 2.

Algorithms for LDS data analyses, including neural networks, were developed and tested, with emphasis on automated star spectral type recognition. The parameters from various LDS plate projects were compared with those of Gaia BR/RP. In addition, various evaluation and visualization techniques have been developed and tested. The potential of Gaia $\mathrm{BP} / \mathrm{RP}$ for astrophysical research was investigated with emphasis on objects with prominent colors and prominent spectral (and variable) spectral features.

\section{Conclusion}

The ESA Gaia satellite will provide ultra-low dispersion spectra by $\mathrm{BP}$ and $\mathrm{RP}$, representing a new challenge for astrophysicists and for computer science. The nearest analogy is digitized prism spectral plates: the Sonneberg, PARI, Hamburg and Byurakan surveys. These digitised surveys can be used for simulation and for tests of the Gaia algorithms and Gaia data. Some algorithms have already been tested. Some types of variable stars are known to exhibit large spectral type changes - however this field is little exploited and more discoveries can be expected with the Gaia data, as Gaia will allow us to investigate the spectral behavior of huge numbers of objects over a period of 5 years with good sampling for spectroscopy. However, the data must first be decontamined to be scientifically applied, as discussed above.

Variability studies based on low dispersion spectra are expected to provide unique novel data, and can use the algorithms recently developed for automatic analyses of digitized spectral Schmidt plates. These variability studies may use either Gaia BP/RP data, or scanned plate data, or both. Then time coverage up to and exceeding 100 years can be achieved. The oldest LDS plates that we have identified in our project are stored at the Carnegie Observatories, Pasadena, CA, USA, and were taken in 1909.

\section{Acknowledgement}

Czech participation in the ESA Gaia project is supported by PECS project 98058. The scientific part of the study is related to grants 205/08/1207 and 102/09/0997, provided by the Grant Agency of the
Czech Republic. Some aspects of the project described here are a natural continuation of Czech participation in ESA INTEGRAL (ESA PECS 98023). The analyses of digitised low dispersion spectral plates are supported by MSMT KONTAKT Project ME09027.

\section{References}

[1] Becker, F.: Spektral-Durchmusterung der Kapteyn-Eichfelder des Südhimmels. I. Pol und Zone -75 deg. Potsdam Publ., 27, 1, 1929.

[2] Henize, K. G.: The Michigan-Mount Wilson southern Ha survey. Astronomical Journal, 59, $325,1954$.

[3] Hudec, L.: Algorithms for spectral classification of stars. BSc. Thesis, Prague : Charles University, 2007.

[4] Jordi, C., Carrasco, J. M.: The Future of Photometric, Spectrophotometric and Polarimetric Standardization, ASP Conference Series, 364, 215,2007 .

[5] Montegriffo, P., et al.: A model for the absolute photometric calibration of GAIA BP and $R P$ spectra. I. Basic concepts. GAIA-C5-TNOABO-PMN-001-1, 2007.

[6] Montegriffo, P.: A model for the absolute photometric calibration of Gaia BP and RP spectra. II. Removing the LSF smearing. GAIA-C5TN-OABO-PMN-002, 2009.

[7] Simon, V., Hudec, R., Pizzichini, G., Masetti, N.: $A \&$ \& $A, \mathbf{3 7 7}, 450,2001$.

[8] Simon, V., Hudec, R., Pizzichini, G., Masetti, N., Gamma-Ray Bursts: 30 Years of Discovery: Gamma-Ray Burst Symposium, AIP Conference Proceedings, 727, 487, 2004a.

[9] Simon, V., Hudec, R., Pizzichini, G.: $A \& 6$, 427, 901, 2004b.

[10] Straizys, V., et al.: Baltic Astronomy, 15, 449, 2006.

[11] Christlieb, N., Wisotzki, L., Grasshoff, G.: $A$ \& A, 391, 397, 2002 .

[12] Hagen, H.-J., et al.: $A \& A S, \mathbf{1 1 1}, 195,1995$.

[13] Fynbo, J., et al.: ApJSS, 285, 526, 2009.

[14] Straižys, V., Lazauskaitè, R., Zdanavičius, K.: Baltic Astronomy, 19, 181, 2010. 
René Hudec

E-mail: rhudec@asu.cas.cz

Astronomical Institute

Academy of Sciences of the Czech Republic

CZ-25165 Ondřejov, Czech Republic

Faculty of Electrical Engineering

Czech Technical University in Prague

Technická 2, CZ-16627 Prague, Czech Republic
Lukáš Hudec

Miloš Klíma

Faculty of Electrical Engineering

Czech Technical University in Prague

Technická 2, CZ-16627 Prague, Czech Republic 